\title{
La psicología de las hormigas en la obra de Ramón y Cajal
}

\author{
Virgili lbarz Serrat \\ Universidad Ramon Llull \\ Barcelona, España
}

\begin{abstract}
Recibido: 5 de julio del 2017 / Aceptado: 20 de julio del 2017 doi: https://doi.org/10.26439/persona2017.n20.1740
\end{abstract}

\begin{abstract}
Santiago Ramón y Cajal expone que, para investigar la psicología de las hormigas, hay que tener en cuenta los datos relativos a los tropismos, percepciones, memoria asociativa, actos reflejos, instintos superiores, etcétera. Divide a las hormigas en dos grandes grupos: hormigas poliópsicas, cuyos ojos poseen ochocientas o más facetas corneales, que ven bien o regularmente; y hormigas oligópsicas, que ven poquísimo y cuyas facetas corneales oscilan entre setenta y quinientas. Cajal concluye que las hormigas oligópsicas tienen una gran pobreza sensorial. Excepto el tacto y el olfato, que alcanzan un gran desarrollo, los demás sentidos aportan a las hormigas confusas y fragmentarias observaciones del mundo exterior. En cambio, las hormigas poliópsicas son sensibles a los colores y se impresionan especialmente con el color negro.
\end{abstract}

hormigas / sensaciones visuales / impresiones olfativas / impresiones táctiles

\section{The Psychology of Ants in the Work of Ramón y Cajal}

Santiago Ramón y Cajal explains that in order to investigate the psychology of ants, we must consider the data related to tropisms, perceptions, associative memory, reflex acts, superior instincts, etc. He divides the ants into two large groups: poliopsic ants, whose eyes have eight hundred or more corneal facets, which see well o regularly, and oligopsic ants that look very little and whose corneal facets range from seventy to five hundred. Cajal concludes that oligopsic ants have a great sensorial poverty. Except tact and smell, which are very developed, the remaining senses render confused and fragmented observations of the outside world. Poliopsic ants, on the other hand, are sensitive to colors and particularly impressed with black.

ants / visual sensations / olfactory impressions / tactile impressions

Correo electrónico: virgiliis@blanquerna.url.edu 


\section{INTRODUCCIÓN}

Santiago Ramón y Cajal, en su casa de campo de Cuatro Caminos de Madrid y cuando le quedaba poco tiempo para jubilarse, se dedicó a estudiar la vida de las hormigas. Este trabajo le fue solicitado por Ignacio Bolívar (1850-1944) para la celebración del cincuentenario de la Sociedad Española de Historia Natural. Fue publicado con el título "Las sensaciones de las hormigas", en 1921. En esta investigación, describe con todo detalle el proceso que sigue para averiguar cómo se producen las sensaciones de las hormigas.

De acuerdo con Ramón y Cajal, para analizar la psicología de las hormigas, hay que tener en cuenta las cuestiones relativas a los tropismos, datos sensoriales, percepciones, memoria asociativa, actos reflejos, instintos superiores, etcétera, que han sido investigados por John Lubbock (1834-1913), Jean-Henri Fabre (1823-1915), Auguste Forel (1848-1931) y Henri Piéron (1881-1964), entre otros. Su labor consistirá en investigar lo que haya de cierto en los trabajos de estos autores, pero sin la certidumbre de lograrlo, ya que son muchas las causas de error.

Los naturalistas estaban de acuerdo en que las hormigas poseen cuatro sentidos fundamentales, base de su vida psíquica: el visual, el olfativo, el táctil y el gustativo. De estos sentidos conocían la porción receptora, pero muy poco los centros del ganglio cerebroide donde la impresión se convierte en sensación. Ramón y Cajal dice que a estos sentidos habría que añadir el acústico, pero a juzgar por la sordera, bien comprobada, de las hormigas, quizás se trate de un órgano rudimentario y de dudosa utilidad. Para él, los otros sentidos otorgados a las hormigas, como el de la dirección, le parecen muy problemáticos. El sentido de la dirección podría interpretarse simplemente como manifestaciones de la memoria de dirección inicial y de los principales incidentes ocurridos en el trayecto.

Ramón y Cajal expone que al mismo proceso psicológico de retentiva de lugares y rutas pertenece la memoria muscular de Piéron, considerada muy importante para la dilucidación del regreso al nido. Precisa que incluso en el hombre, donde la memoria inconsciente alcanza su plenitud, ayudada por aparatos receptores complicados, no nos orienta eficazmente. La memoria inconsciente solo nos permite la medición automática y no siempre exacta de la cantidad de movimiento necesario para remontar en la oscuridad una escalera, reconocer la posición de un mueble o el sitio aproximado de un timbre.

Y si esto ocurre en los animales superiores, ¿cómo admitir dicho sentido, con fines de infalible orientación hasta por terrenos desconocidos, en la hormiga, en cuyos músculos y tendones nadie ha logrado encontrar algo comparable a los husos de Kühne (estación del sentido muscular) o los órganos músculo-tendíneos de Golgi? (Ramón y Cajal, 1921a, p. 557) 


\section{Clasificación SEnSORIAL DE LAS HORMIGAS}

Piéron clasificó a las hormigas en tres grupos sensoriales: visual, olfativo y muscular. Ramón y Cajal expone que adoptaría de buen grado la clasificación de Piéron, si no fuera porque la admisión de tres grupos sensoriales choca en la práctica con serias dificultades. Considera que es más sencillo clasificar a las hormigas en solo dos grupos: las que ven bien o regularmente (poliópsicas), cuyos ojos poseen ochocientas o más facetas corneales, y las que ven poquísimo (oligópsicas), cuyas facetas corneales oscilan entre setenta y quinientas.

Para Ramón y Cajal, al estudiar las sensaciones de las hormigas, se producen errores por no haber tenido en cuenta la influencia perturbadora de ciertos estados psicológicos actuales o preexistentes, que modifican los resultados obtenidos. Expone que, en primer término, cada hormiga posee cierta "individualidad psíquica". En segundo lugar, hemos de tener en cuenta el fenómeno del "ensimismamiento o distracción", muy común en las hormigas obreras cargadas y en las absorbidas en la construcción del nido. En tercer lugar, tenemos el "estado emocional" de las hormigas producido por la imposición de las condiciones artificiales de la experimentación en pugna con los hábitos arraigados. Explica lo siguiente:

Para la interpretación de algunos hechos negativos, que a primera vista parecen implicar extremada penuria de ciertos sentidos, conviene tener presente que la hormiga suele guiarse en sus labores por la impresión sensorial dominante [cursivas del autor]. Este comportamiento representa un ahorro de esfuerzo nervioso. Condúcese, pues, como nosotros, que para orientarnos bien lo fiamos todo a la vista, desdeñando o inadvirtiendo las impresiones táctiles, olfativas y la sensibilidad a las vibraciones mecánicas; impresiones de capital importancia, según es notorio, en la marcha de los ciegos. (Ramón y Cajal, 1921a, p. 559)

\section{Sensaciones Visuales}

Lubbock y otros observadores admitían que las hormigas percibían los colores. Para Ramón y Cajal, este hecho no estaba demostrado. Lo que se deducía de los experimentos de Lubbock no es que las hormigas discriminen los colores, sino que son afectadas, al modo de la placa fotográfica, por las radiaciones más breves del espectro, es decir, las dotadas de mayor poder fotoquímico.

Ramón y Cajal sentía un gran interés por la anatomía del ojo de los insectos. Entre sus trabajos destacan "Nota sobre la estructura de la retina de la mosca" (1909), "Plan fundamental de la retina de los insectos" (1915), "Observaciones sobre la estructura de los ocelos y vías nerviosas ocelares de algunos insectos" (1918) y "Sobre la estructura de los centros ópticos de los insectos" (1921b).

Considera que es muy difícil estudiar la anatomía del ojo de las hormigas oligópsicas a causa de la enorme dificultad 
para obtener cortes finos bien teñidos del aparato ocular. Las preparaciones del ojo de las hormigas Aphaenogaster, Camponotus cruentatus, etcétera, muestran inmediatamente detrás de las corneolas biconvexas una capa compacta y continua de pigmento pardo-negro, que absorbe totalmente las radiaciones espectrales. Por eso, resulta muy difícil comprender cómo una imagen coloreada podría impresionar cualitativamente las células receptoras y producir, según debe ocurrir en los insectos de visión lúcida (abejas, avispas, tábanos, mariposas, mosca azul, etcétera), fenómenos fotoquímicos específicos generadores de corrientes nerviosas.

Para Ramón y Cajal, es muy probable que las hormigas no disciernan los colores. Todos sus experimentos lo confirman. El espacio visual percibido por estos insectos se puede imaginar como un panorama nebuloso, donde destacan solamente algunos objetos próximos de gran tamaño. Cita sus experimentos que prueban que las hormigas oligópsicas carecen de la visión de los colores. Estos insectos no revelan el menor signo de sorpresa o extrañeza cuando, de regreso de sus excursiones, encuentran las pistas o las aberturas del nido teñidas intensamente con diversos colores de anilina. Se advierte la misma indiferencia cuando delante de las hormigas obreras en marcha son proyectadas las radiaciones del prisma o la luz solar tamizada por cristales coloreados.

Incluso las hormigas poliópsicas, como la Lasius niger, no siente repulsión en presencia de una gota de miel o de mermelada pintadas con eosina o azul de metileno. Ramón y Cajal dice que, en cuanto a los alimentos, el criterio supremo de la hormiga es el sabor. Lubbock y Forel creían que las hormigas oligópsicas eran sensibles a los rayos ultravioletas. Ramón y Cajal lo pone en duda. Reproduce los experimentos de Lubbock y Forel usando un aparato con electrodos de magnesio y de cubetas cubiertas por portaobjetos de cuarzo. Para él, las hormigas oligópsicas Camponotus cruentatus, Aphaenogaster, Tapinoma, etcétera, evitan (no siempre) la acción de las radiaciones ultravioletas, pero no a causa de la percepción cromática (efecto cualitativo de ondas muy breves), sino por su acción irritante sobre el cuerpo de la hormiga y quizás sobre los pelos táctiles.

\section{Distinción de la luz y la sombra}

Las hormigas oligópsicas (Tapinoma erraticum, Aphaenogaster barbara, Pheidole megacephala, etcétera) son incapaces de distinguir cualitativamente las diversas longitudes de onda del espectro, pero diferencian bastante bien la luz de la sombra, con tal de que el contraste sea bastante acentuado.

Ramón y Cajal expone uno de sus experimentos: si a una hormiga se le obliga a pasar por un largo túnel de cartón dispuesto en forma de bóveda, no vacila cuando el trayecto es corto; pero muchas se asustan y retroceden cuando el túnel mide de 8 a 10 centímetros de longitud. Tampoco distinguen un cristal puesto a 3 o 4 milímetros del suelo sobre la ruta: casi todas las hormigas pasan por debajo hasta 
llegar a un punto en que por la exigüidad del espacio vertical chocan contra el vidrio. Entonces viran casi siempre en ángulo recto en busca de los bordes, para emerger y bordear el obstáculo.

Las hormigas diferencian lo tenebroso de lo claro, como muestra la curiosidad de las Aphaenogaster barbara, las Pheidole, etcétera. Se acercan a un terrón de azúcar puesto en la vecindad de la ruta, a condición de que la hormiga pase a menos de medio centímetro de la golosina. Sin embargo, para Ramón y Cajal, este hecho perceptivo no siempre es fácil de comprobar en las hormigas oligópsicas. Considera que si la hormiga regresa cargada o arrastra tierra arrancada del suelo, suele ser indiferente a los cebos más apetitosos por la distracción o ensimismamiento.

Otra prueba de la impresionabilidad la ofrece el fenómeno del deslumbramiento. Si con una lente se concentra la luz sobre una pista - sin elevar demasiado la temperatura-, las hormigas se asustan; muchas retroceden después de penetrar en el foco; algunas dan un rodeo, acabando por orientarse, y solo las que van muy cargadas, y, por tanto, en estado de profunda distracción, cruzan impasibles el foco luminoso.

En cambio, las hormigas poliópsicas, como Myrmecocystus viaticus y Lasius niger, reaccionan mucho más vivamente a los focos lenticulares. En estas hormigas, según era de presumir, el fenómeno de deslumbramiento se desarrolla más fácil y rápidamente. Se advierten titubeos e inquietudes en presencia de la luz solar reflejada por espejos. A pesar de ello, no se despistan y cruzan cautelosamente la zona iluminada, o dan un rodeo con precaución, y acaban por incorporarse a la ruta de sus compañeras y llegan hasta el nido.

\section{Acuidad visual de las hormigas}

Por regla general, y salvando excepciones, los objetos muy delgados y plantados verticalmente en la pista (de 0,5 a 1 centímetro) y separados verticalmente no son percibidos. Las hormigas oligópsicas distinguen el obstáculo imprevisto cuando lo tocan con las antenas. La mayor o menor facilidad de la travesía de vallas y enrejados depende de la amplitud de los espacios. Con enrejados cuyos alambres están separados unos 4 milímetros pasan la mayoría de las obreras Tapinoma y Aphaenogaster; con enrejados de 3 milímetros disminuye notablemente el número, salvando la alambrada las obreras medianas y pequeñas; un enrejado de 2 milímetros constituye un obstáculo infranqueable. Al topar con el obstáculo, casi todas las hormigas se desvían en ángulo recto, bordean la tela metálica y ensayan el paso por parajes más alejados; algunas retroceden descorazonadas, y otras flanquean lateralmente el enrejado, incorporándose a la pista, después de dar un gran rodeo. Para Ramón y Cajal (1921a):

Este espectáculo sugiere, más que una visión confusa de las vallas, la de una filtración o tamización, exclusivamente regulada por el tamaño de las obreras y la envergadura de las antenas; envergadura variable, naturalmente, en cada hormiga, a causa del diverso grado de aducción o inclinación hacia adelante de tales apéndices. (p. 564) 
Las hormigas oligópsicas Aphaenogaster, Camponotus cruentatus y Tapinoma se comportan como si estuvieran totalmente ciegas y se guiaran exclusivamente por el tacto y el olfato. La acuidad visual es mucho mayor en las hormigas poliópsicas. La Myrmecocystus viaticus suele atisbar los enrejados puestos verticalmente delante del nido, a una distancia de 1 y hasta 2 centímetros. Se puede ver a las obreras que regresan al nido rodear el obstáculo sin tocarlo, para incorporarse al hormiguero, y a las atareadas con la labor de extracción de escombros evitar la empalizada, marchando en otra dirección.

Las observaciones de Ramón y Cajal revelaron que las hormigas poliópsicas se impresionan especialmente del color negro, con tal de que despida reflejos brillantes. En tales circunstancias, la acuidad visual aumenta notablemente. Cita dos ejemplos:

Una comunidad de Lasius niger (variedad provista de ocelos y de ojos de finas facetas) invadió nuestra casita de campo, haciendo nido en las grietas del embaldosado. A distancia de varios metros, y no lejos de amplia pista recorrida por obreras exploradoras, pusimos sobre un cristal negro tres pequeñas gotas de materias mucilaginosas de brillantes reflejos marginales: una de las gotas era de miel, otra de goma arábiga $\mathrm{y}$, en fin, otra de cola de comercio. Todas tres mostraban sensiblemente igual matiz amarillento, casi imperceptible sobre fondo oscuro. (Ramón y Cajal, 1921a, p. 565)

A los pocos minutos, observó que algunas hormigas repararon en el botín, estableciéndose una pista desde el nido a las gotas. Con sorpresa, advirtió que las tres gotas atrajeron por igual a las hormigas. Conforme suponía, las posadas al borde de la miel aumentaron progresivamente. Engolosinadas por la primera libación, visitaron reiteradamente el cebo; mientras que las hormigas empeñadas en saborear la goma arábiga y la cola antiséptica quedaban prendidas, sin poder repetir sus expediciones. A Ramón y Cajal le parece indudable que lo que atrajo imperiosamente a la comunidad de Lasius niger no fue el olor ni el color, sino el vivo reflejo luminoso de las sustancias mucilaginosas, percibido de 1 a 0,5 centímetros de distancia.

Otro ejemplo de la capacidad de distinguir objetos diminutos oscuros con tal de que brillen mucho lo ofrecen las hormigas cazadoras, aunque pertenezcan al grupo de las oligópsicas:

Varias obreras de Aphaenogaster barbara fueron asfixiadas por el cloroformo, tratadas subsiguientemente, primero con el alcohol y el éter, y después por diversos agentes alcalinos, a fin de eliminar en lo posible el olor fórmico; finalmente, se desecaron al sol durante una semana. En tal estado de momificación, abandonáronse en las inmediaciones de nidos de Aphaenogaster testaceopilosa y de Myrmecocystus viaticus. Incontinenti fueron atisbadas por obreras exploradoras que, considerándolas como excelente botín, las condujeron a los silos subterráneos. (Ramón y Cajal, 1921a, p. 565)

Ramón y Cajal precisa que, si antes de ser emplazadas las Aphaenogaster barbara en la vecindad de los hormi- 
gueros se las pintaba de blanco, las hormigas cazadoras pasaban a su lado sin reconocerlas. Es una prueba inequívoca de que el color negro brillante propio de muchos insectos les impresiona mucho más que la forma y el olor, y de que las hormigas de poca visión aciertan a distinguir de cerca un objeto negro de 2 o 3 milímetros de diámetro. Se obtuvieron los mismos resultados con los cadáveres de hormigas que sus compañeras de comunidad extrajeron del nido, guiadas por un instinto higiénico. Pintados de varios colores fueron inadvertidos.

Para el médico español, las percepciones visuales influyen muy poco en la vida de las hormigas oligópsicas. Cita el hecho, bien conocido y comprobado por él, de que la Camponotus aethiops y la Aphaenogaster barbara trabajan lo mismo de día que de noche, y con igual diligencia y brío durante las noches frías que en las noches claras de verano.

Un experimento muy practicado consistía en la obstrucción de los ojos de las hormigas mediante barnices opacos para la cauterización de las corneolas. Ramón y Cajal reproduce este experimento, pero la interpretación de los resultados le parece muy ardua. Considera que todo insecto cegado se desconcierta y queda como desmoralizado y alocado. Por exigua que sea la ventana cerrada al mundo exterior, constituye para la hormiga una fuente preciosa de informaciones, complementarias a las aportadas por los sentidos olfativo $\mathrm{y}$ táctil. Además, es difícil señalar la parte en que en tal desorientación toman, respectivamente, la supresión de la imagen luminosa y el deterioro o empaste de algunos pelos táctiles cefálicos y, sobre todo, la emoción del animal al sentirse primero amarrado y después libre sobre una pista cuya dirección ha olvidado.

$\mathrm{Si}$ hemos de dar crédito a sus observaciones, el atolondramiento de las hormigas obreras y soldados ciegos es mucho menor que el producido por la sección de las antenas. Con un poco de paciencia, consiguió sorprender en el jardín de su casa algunas hormigas oligópsicas Camponotus, Tapinoma y Aphaenogaster. Después de ennegrecer sus ojos con betún de Judea, las dejó retornar al nido a tientas, y después de mucho tiempo de estupor y extravío, y de reiteradas tentativas para limpiarse las corneolas.

\section{IMPRESIONES OLFATIVAS}

Ramón y Cajal siente un gran interés por las sensaciones visuales, pero también analiza las impresiones olfativas y táctiles de las hormigas. En 1915, había publicado "Contribución al conocimiento de los centros nerviosos de los insectos", junto con Domingo Sánchez. Los naturalistas admitían en las hormigas un sentido olfativo exquisito. Las observaciones del médico español en el jardín de su casa de verano confirmaron las de los especialistas en las hormigas. Para Ramón y Cajal, de negar dicha sensibilidad, resultaría muy difícil la comprensión de algunos actos de las hormigas oligópsicas.

Los puntos por examinar y establecer sobre esta materia son la distancia a la que las hormigas oligópsicas y 
poliópsicas huelen; si su escala odorífera se corresponde con la nuestra y la de los mamíferos superiores, y averiguar si en sus trabajos, cacerías y expediciones el sentido olfativo goza de alguna preponderancia sobre los demás. Para Ramón y Cajal, la mayoría de los olores fuertes, agradables o nauseabundos, percibidos por nosotros, lo son también por las obreras oligópsicas:

El olor a alcanfor, el del amoniaco, de la trementina, de la asafétida, de la piridina, del timol, del creosol, de las esencias de clavo, bergamota, de anís y de orégano, del éter, del alcohol amílico y alílico, etc., las impresiona enérgicamente, produciéndoles una repugnancia invencible. Regla general: la hormiga huye alarmada de toda emanación odorífera a que no está habituada. (Ramón y Cajal, 1921a, p. 567)

La distancia de impresión, el "dintel de la excitación", varía para cada especie. Normalmente es muy corta, lo que se explica bien por la ausencia de aparato colectory conservador de las emanaciones olfativas. Cajal ha observado que la Aphaenogaster barbara, la Pheidole, la Tapinoma, etcétera, retroceden ante una mancha de piridina, de esencia de clavo o de bergamota situadas entre uno y medio centímetro. Aunque por la distracción hay obreras sobrecargadas que llegan hasta el borde de la mancha, pocas veces el ímpetu les lleva a penetrar en el trozo de pista empapada de la esencia.

Hay hormigas poliópsicas, como la Myrmecocystus viaticus, que reconocen los olores desagradables a mayor distancia que las oligópsicas. Observó que, derramadas varias gotas de bergamota en torno al nido, ninguna de las obreras cargadas de escombros se atrevió a salir, a pesar de que entre la abertura del nido y el círculo oloroso había más de 2 centímetros. Las hormigas que regresaban de las expediciones retrocedieron a distancias variables entre 1,5 y 3 centímetros. Los olores demasiado persistentes, como el de la esencia de clavo arrojada en la pista, obligan a ciertas especies de hormigas a abandonar el antiguo camino y a trazar uno nuevo, paralelo al anterior.

Ramón y Cajal piensa que en estos experimentos se analiza la acción de olores insólitos y desagradables o incómodos para las hormigas. Pero pudiera ocurrir que las emanaciones que excitan su sensibilidad y motivan sus reacciones fueran totalmente inaccesibles para nosotros. La escala olfativa por la cual se guían pudiera coincidir solo parcialmente con la nuestra. Esta disparidad de escalas olorosas se advierte ya entre los mamíferos. El perro percibe olores absolutamente imperceptibles para nosotros. Cita un ejemplo referente a la hormiga amazona, de la que poseía un populoso nido en su jardín. Había observado muchas veces que la columna de la Polyergus rufescens se detenía bruscamente al llegar a un montón de escombros, de sarmiento o de broza; una vez allí:

Sin la menor vacilación, precipítanse todos los asaltantes en los intersticios y recovecos del laberinto de ladrillos y malezas, de donde emergen, a los pocos minutos, prendidas en los garfios 
mandibulares, larvas y crisálidas pertenecientes a la tímida F. rufibarbis. Ahora bien: la exploración escrupulosa del montón de escombros donde penetraron las feroces amazonas no permitió observar el menor indicio del hormiguero, ni descubrir tampoco obreras dispersas en busca de botín. Solo al final del desastre asomó tal cual rufibarbis, con intención, sin duda, de salvar algún hijuelo, por azar abandonado por los invasores. (Ramón y Cajal, 1921a, p. 569)

Ramón y Cajal dice que se ha exagerado mucho la importancia que en la orientación de las hormigas oligópsicas posee el sentido olfativo. Cree que en el reconocimiento de las pistas colaboran también diversos datos sensoriales, y muy especialmente la impresión de los pelos táctiles. En apoyo de su idea, recuerda que, cuando las hormigas cargadas se desvían por un accidente imprevisto (golpe de viento, por ejemplo), muchas de ellas cruzan su pista o se acercan al nido sin reconocerlo, por lo menos, durante el primer cuarto de hora.

\section{IMPRESIONES TÁCTILES}

Para Ramón y Cajal, la importancia de las impresiones táctiles para las hormigas oligópsicas se impone con solo examinar la cantidad prodigiosa de pelos largos y cortos que erizan las antenas, la cabeza y sobre todo las patas. En el bulbo terminal de las antenas de Tapinoma y Aphaenogaster barbara, los apéndices son tan abundantes que en algunos parajes casi tocan las criptas de donde emergen. Para los efectos de la marcha, las impresiones táctiles dominantes son recogidas por los garfios córneos y pelos de las patas.

Cree que es muy fácil demostrar experimentalmente la sensibilidad táctil de las hormigas. Es suficiente con alisar o cambiar ligeramente, por medios mecánicos, el suelo de las pistas, o cubrir las pistas con tules o enrejados que, dejando a salvo las emanaciones olfativas, transformen el relieve. Ante enrejados finos o anchas mallas emplazados horizontalmente sobre las pistas, casi todas las hormigas obreras sufren grandes perturbaciones, a pesar de percibir el olor específico. Las que van cargadas avanzan titubeando y deteniéndose a cada paso, pero la mayoría no atraviesa el inesperado obstáculo, o si lo recorren en una brevísima extensión, es para torcer en ángulo recto y ganar la orilla. Por lo general, cuanto más fino es el retículo, mayor es la sorpresa y desorientación.

Las desviaciones observadas en las hormigas mediante la colocación de retículos sobre la pista o junto al nido suelen ser mayores que las sufridas por los experimentos de barrido, irrigación o deformación de los caminos mediante erosiones o colocación de tierra. Si sobre un pequeño trozo de pista se superpone tierra superficial tomada mediante una espátula delgada de otro segmento de la misma pista, la desorientación en la hormiga Aphaenogaster barbara es completa, a pesar de la persistencia del mismo olor. Ramón y Cajal (1921a) expone:

Sin negar que en los resultados obtenidos por numerosos sabios influya algo la atenuación o descarte de tales efluvios, 
juzgamos que la causa desorientadora principal consiste en la modificación del relieve del suelo, del que la hormiga conserva memoria fidelísima. Y aun seríamos más afirmativos y categóricos si no fuera notorio que sobre la corteza de los árboles siguen fidelísimamente ciertas pistas preestablecidas (Lasius y Tapinoma). (p. 570)

Para el médico español, en estos mismos ejemplos, no parece desdeñable el papel desempeñado por las impresiones táctiles, conforme lo prueba la preferencia de las hormigas por ciertas resquebrajaduras profundas de la corteza de los árboles, muy ricas en referencias estereoscópicas, y la desorientación e inquietud que sufren al cruzar por un segmento cortical suavizado y pulido por un cuerpo duro (mango de bastón, espátula de marfil, etcétera), o ligeramente empastado por un color transparente.

Considera que como anejos del sentido táctil se pueden incluir la capacidad bien conocida de las hormigas de apreciar contrastes de temperatura (sentido térmico) y las excitaciones dolorosas (sentido del dolor). Piensa que es muy posible que, al modo que sucede en los mamíferos, la piel de las hormigas disponga de nervios específicos térmicos, dolorosos y táctiles, distribuidos en zonas diferentes, es decir, en apéndices pilosos. Pero precisa que sus experimentos acerca de este punto distan mucho de estar acabados.

\section{INTERPRETACIÓN PSICOLÓGICA}

Para Ramón y Cajal, las hormigas oligópsicas adolecen de una gran penuria sensorial. Excepto el tacto y el olfato, los demás sentidos aportan confusas y fragmentarias informaciones del mundo exterior. Insensibles a los colores, incapaces de la percepción del relieve, distinguen solamente, a pequeñísimas distancias y sin detalles, objetos de gran tamaño relativo, olfatean desde muy cerca, no tienen un aparato colector de los olores, carecen casi enteramente de oído y aprecian solo variaciones térmicas de muchos grados. En este aspecto, se puede apreciar la gran valoración cajaliana del cerebro: es la idea de la dialéctica cerebro-sentidos que enriquece la perspectiva evolucionista. El cerebro, con su intencionalidad, manda a los sentidos que se le subordinan:

Como en todas las especies animales, el mundo exterior percibido por la hormiga es un mundo aparte, específico, fundamentalmente diferente del nuestro, excepto la percepción de ciertas propiedades geométricas y de determinadas emanaciones materiales.

Y, no obstante esta pobreza sensorial, dichos insectos despliegan, por compensación, un lujo prodigioso de reacciones motrices y de instintos de finalidad maravillosa. $\mathrm{Y}$ es que los sentidos no son lo más importante de la vida psíquica: por encima de ellos, coordinando sus datos e interpretándolos a la luz de las milenarias adquisiciones de la especie, impera el cerebro, riquísimo en potencialidades. (Ramón y Cajal, 1921a, p. 571)

Pero no se detiene en una posición positivista estricta. Influenciado por el 
romanticismo, establece comparaciones entre las hormigas oligópsicas y los sordomudos y ciegos de nacimiento:

Yo compararía de buen grado a los ciegos y sordomudos de nacimiento, de que son ejemplos admirables Laura Bridgman — que además de ciega y sorda carecía de gusto y olfato- y la célebre Helen Keller. Ambas, y singularmente la última, sin más recurso sensorial que el tacto, sabia y metódicamente educado, lograron desarrollar prodigiosas aptitudes intelectuales innatas, durmientes y como en estado potencial. Hellen Keller, auxiliada por el alfabeto táctil, aprendió a leer, siguió brillantemente una carrera, dominó varios idiomas y escribió libros admirables, donde campean, con la más selecta y copiosa erudición, el más sano y elevado criterio. Al leer sus obras, como la de otros ciegos ilustres, acude a la memoria la frase gráfica de Villey: "La vista es el sentido de las distracciones". (Ramón y Cajal, 1921a, pp. 571-572)

Ramón y Cajal piensa que si nuestros sentidos aportan noticias del mundo exterior, su misión principal consiste en obrar como despertadores de nuestro maravilloso mundo interior. Nuestros sentidos ponen en marcha los instintos superiores y los innatos y complicados mecanismos mnemónicos, sentimentales, representativos y lógicos, valiosísimo legado de la evolución filogenética. Y precisa:

Muy clarividente y acertado mostrose, por consiguiente, Leibniz cuando, corrigiendo el escueto e incompleto aforismo de Locke, nihil est in inte- llectu quod prius non fuerit in sensu ${ }^{1}$, añadió: nisi intellectus ipse ${ }^{2}$. Cabe, pues, disponer de un cerebro poderoso y hasta genial, asistido de mezquinos e incompletos sentidos. (Ramón y Cajal, 1921a, p. 572)

El médico español dice que no pretende identificar el magnífico cerebro humano con el precario ganglio cerebroide de las hormigas. Sus estudios sobre el sistema nervioso central de las hormigas y las moscas revelaron la existencia de una máquina asociativa compleja y sutil. En las hormigas se da en pequeño algo de lo ocurrido con ciertos ciegos y sordomudos: compensan la miseria sensorial con una rica y fina organización del órgano encefálico.

Bajo este aspecto, es muy instructivo comparar los lúcidos y complejos instintos industriales de la hormiga, casi ciega, con la precaria mentalidad de aquellos insectos que, como la mosca, la libélula o la mariposa, están dotados de ojos magníficos, de olfato y tacto exquisito y de vuelo poderoso: "Diríase que la naturaleza, como si tuviera conciencia de sus propias injusticias, se complace a menudo en prodigar todos los dones del espíritu a los más humildes seres, por igual abandonados de la fuerza, de la belleza y de la gracia” (Ramón y Cajal, 1921a, p. 572).

1 Nada hay en el intelecto que antes no haya pasado por los sentidos.

2 Ni siquiera el propio concepto de intelecto. 


\section{Observaciones finales}

La motivación filosófica de la psicología cajaliana es la filosofía materialista formulada por primera vez en Jonia. Los presocráticos, posteriormente Epicuro y más tarde Hipócrates, el padre de la medicina, sostenían que el sentir, el percibir y el pensar eran funciones materiales $\mathrm{y}$, por tanto, funciones del cerebro. Este punto de vista acerca de la naturaleza de la mente forma parte de una ontología naturalista. La actitud positiva del naturalismo, el mecanicismo y el evolucionismo son los fundamentos de las concepciones psicológicas de Ramón y Cajal. Los aspectos esenciales de la psicología cajaliana se basan en las leyes que rigen la morfología y conexión de las células nerviosas de la sustancia gris.

Sin embargo, el idealismo y el romanticismo matizan las concepciones psicológicas cajalianas. En su obra se produce una colisión entre las posiciones positivistas e idealistas. Hemos visto que el romanticismo e incluso el sentimentalismo aparecen en las conclusiones cajalianas sobre la psicología de las hormigas. Ramón y Cajal busca en las hormigas algunas de las facultades mentales de los hombres. Debemos tener en cuenta que este antropomorfismo se encuentra en muchos científicos del siglo XIX. La perspectiva histórica nos hace ver que, aunque la observación y experimentación sobre las hormigas es muy minuciosa, Ramón y Cajal no controla unas variables o factores bien identificados.
Creemos que el médico español está influido por la psicología animal que empieza a desarrollarse a partir de las teorías darwinianas. Esta psicología se caracteriza por la búsqueda de las facultades mentales del hombre en los animales. Se tiene la esperanza de encontrar en ellos las facultades mentales humanas, pero en menor grado. Algunos de los autores más representativos son John Lubbock, Robert Yerkes, Karl Carus y especialmente George John Romanes (1848-1894), fundador de la psicología comparada, basada en las similitudes entre los procesos cognitivos humanos y animales.

Romanes, darwiniano ortodoxo, publica una serie de obras sobre la inteligencia, el pensamiento y el lenguaje de los animales. Una de las obras de este autor, L'intélligence des animaux (1887), se conserva en el Instituto Cajal de Madrid. Los primeros autores insisten en la continuidad entre el hombre y los animales. Sus observaciones están bien hechas e influyen en la historia de la psicología, ya que son los iniciadores de la psicología animal. Sin embargo, estos científicos solo hacían observaciones sobre el comportamiento de los animales y la vida de los insectos, lo cual llevó a la psicología animal a una situación sin salida.

\section{RefERENCIAS}

Ramón y Cajal, S. (1909). Nota sobre la estructura de la retina de la mosca. Trabajos del Laboratorio de Investigaciones Biológicas de la Universidad de Madrid, 7, 217-257. 
Ramón y Cajal, S. (1915). Plan fundamental de la retina de los insectos. Boletín de la Sociedad Española de Biología, 4, 105-115.

Ramón y Cajal, S. (1918). Observaciones sobre la estructura de los ocelos y vías nerviosas ocelares de algunos insectos. Trabajos del Laboratorio de Investigaciones Biológicas de la Universidad de Madrid, 16, 109-139.

Ramón y Cajal, S. (1921a). Las sensaciones de las hormigas. En Real Sociedad Española de Historia Natural, Volumen extraordinario con motivo del $50 .^{\circ}$ aniversario de su fundación (pp. 555-572). Madrid: Museo Nacional de Ciencias Naturales.

Ramón y Cajal, S. (1921b). Sobre la estructura de los centros ópticos de los insectos. Revista Chilena de Historia Natural, 25, 1-18.

Ramón y Cajal, S., y Sánchez, D. (1915). Contribución al conocimiento de los centros nerviosos de los insectos. Trabajos del Laboratorio de Investigaciones Biológicas de la Universidad de Madrid, 13, 1-161.

Romanes, G. J. (1887). L'intelligence des animaux. París: Félix Alcan. 
